\title{
A novel role for constitutively expressed epithelial-derived chemokines as antibacterial peptides in the intestinal mucosa
}

\author{
K Kotarsky ${ }^{1}$, KM Sitnik ${ }^{1}$, H Stenstad ${ }^{1}$, H Kotarsky ${ }^{2}$, A Schmidtchen ${ }^{3}$, M Koslowski ${ }^{4}$, J Wehkamp ${ }^{4}$ and \\ WW Agace ${ }^{1}$
}

Intestinal-derived chemokines have a central role in orchestrating immune cell influx into the normal and inflamed intestine. Here, we identify the chemokine CCL6 as one of the most abundant chemokines constitutively expressed by both murine small intestinal and colonic epithelial cells. CCL6 protein localized to crypt epithelial cells, was detected in the gut lumen and reached high concentrations at the mucosal surface. Its expression was further enhanced in the small intestine following in vivo administration of LPS or after stimulation of the small intestinal epithelial cell line, $\mathrm{mIC}_{\mathrm{c} 12}$, with IFN $\gamma$, IL-4 or TNF $\alpha$. Recombinant- and intestinal-derived CCL6 bound to a subset of the intestinal microflora and displayed antibacterial activity. Finally, the human homologs to CCL6, CCL14 and CCL15 were also constitutively expressed at high levels in human intestinal epithelium, were further enhanced in inflammatory bowel disease and displayed similar antibacterial activity. These findings identify a novel role for constitutively expressed, epithelial-derived chemokines as antimicrobial peptides in the intestinal mucosa.

\section{INTRODUCTION}

Intestinal microbes contribute to the well being of the host in several important ways including food degradation, vitamin synthesis, prevention of gut colonization by potential pathogenic organisms, and immune cell development ${ }^{1}$ (for reviews $\left.\mathrm{see}^{2,3}\right)$. However, intestinal bacteria also represent a potential risk to the host in compromised epithelial barrier function and ensuing infection that must be counteracted by innate defense mechanisms at the mucosal surface. Epithelial-expressed antimicrobial peptides (AMPs $)^{4-6}$ represent an important component of this innate defense. These positively charged peptides are secreted into the intestinal lumen and bind to negatively charged microbial membranes; ${ }^{5}$ inducing membrane permeabilization and leakage of bacterial cell content. ${ }^{5,7}$

The importance of AMPs in maintaining gut homeostasis is evident from observations in mice that express human $\alpha$ defensin HD-5 under an intestinal specific epithelial promotor that display decreased susceptibility to Salmonella infection. ${ }^{4}$ Notably, mutations in the NOD-2 gene, that encodes the intracellular receptor for bacterial muramyldipeptide, leads to reduced expression of epithelial-derived AMPs in vitro and in vivo.$^{8-10}$ As approximately a third of all Crohn's patients harbor mutations in the NOD- 2 gene $^{11}$ reduced bacterial clearance as a result of reduced epithelial AMP expression has been implicated as a contributing factor in this disease. ${ }^{9}$ Based on these findings, a detailed analysis of intestinal AMPs in health and disease is clearly warranted.

Chemokines are low molecular weight proteins $(7-16 \mathrm{kDa})$ primarily recognized for their role in the regulation of immune cell migration, including the recruitment of leukocyte subsets to intestinal tissues. ${ }^{12-16}$ In addition, a subset of chemokines display antimicrobial properties. ${ }^{17-19}$ Several antimicrobial chemokines have been shown to be expressed at epithelial surfaces. Examples include CXCL14 in the skin, ${ }^{20}$ CXCL9 and CXCL10 in tonsil fluid ${ }^{21}$ and CCL28 in salivary glands. ${ }^{22}$ However, despite these findings little is known regarding the expression and role of anti-microbial chemokines in the intestinal mucosa.

Here, we identify a subgroup of chemokines, murine CCL6, and its human homologs CCL14 and CCL15, that are constitutively and highly expressed by intestinal epithelial cells and

\footnotetext{
${ }^{1}$ Immunology Section, Department of Experimental Medical Sciences, Lund University, Lund, Sweden. ${ }^{2}$ Division of Pediatrics, Department of Clinical Sciences, Lund University, Lund, Sweden. '3ivision of Dermatology and Venereology, Department of Clinical Sciences, Lund University, Lund, Sweden. ${ }^{4}$ Institute of Clinical Pharmacology, Stuttgart, Germany. Correspondence: K Kotarsky (Knut.Kotarsky@med.lu.se)

Received 18 February 2009; accepted 26 August 2009; published online 7 October 2009. doi:10.1038/mi.2009.115
} 
that display antibacterial activity at concentrations present at the intestinal surface. These results identify a novel role for epithelial-derived chemokines as AMPs in the gut mucosa.

\section{RESULTS}

Chemokine expression by murine intestinal epithelial cells To identify chemokines that are constitutively expressed by murine intestinal epithelial cells, RNA was prepared from lasercaptured intestinal epithelial cells from conventional and germfree mice as previously described ${ }^{23}$ and probed to a gene chip. Intraepithelial lymphocytes, which were readily identified on $\mathrm{H}$ and $\mathrm{E}$ sections, were destroyed before capture, to prevent RNA contamination from these cells. Efficient removal of intraepithelial lymphocytes was confirmed by the absence of CD3, CD8 $\alpha$ and CD45 transcripts in the array data (data not shown). Ten chemokine transcripts were identified as present in at least one of the samples (Figure 1a). Several of these chemokines, including CCL25 $5^{14,23,24}$ and $\mathrm{CX}_{3} \mathrm{CL}^{25,26}$ have been reported to be constitutively expressed by murine intestinal epithelial cells and thus serve as valuable controls. Surprisingly, one of the highest expressed chemokines from both conventional and germ-free mice was CCL6 (Figure 1a), the expression of which was not previously reported in intestinal epithelial cells.

\section{Expression of CCL6 in the intestinal mucosa}

To confirm CCL6 expression in the intestinal mucosa, CCL6 mRNA levels were determined in intestinal and non-intestinal tissues by qRT-PCR (Figure 1b). Although CCL6 was expressed in a number of tissues, including the lung, as reported, ${ }^{27,28}$ highest expression was observed in intestinal tissues (Figure 1b). Similar results were obtained using NCBI's Unigene expression database (CCL6 $=$ Mm.137) that quantifies gene expression based on number of expressed sequence tags (Supplementary Figure 1A online). When comparing CCL6 transcript level to that of CCL25 and $\mathrm{CX}_{3}$ CL1, CCL25 was the most highly expressed small intestinal chemokine, followed by CCL6. Notably, CCL6 mRNA was by far the most abundant chemokine transcript expressed by colonic epithelial cells (Supplementary Figure 1B online). Expression of CCL6 protein was confirmed by western blot (Supplementary Figure 1C online) and immunohistochemical staining showed CCL6 expression primarily in intestinal crypts (Figure 1c) on the apical side of epithelial cells, indicating that CCL6 maybe secreted into the lumen. Consistent with this possibility, CCL6 was readily detected in luminal washes by ELISA (Figure 1d).

To determine whether epithelial cells are the major source of intestinal CCL6, CCL6 mRNA expression was compared in intestinal epithelial and lamina propria (LP) fractions by qRTPCR (Figure 1e). Lack of intestinal alkaline phosphatase mRNA confirmed the absence of epithelial cells in LP preparations (data not shown). Although CCL6 mRNA was present at high levels in epithelial preparations, it was barely detected in the LP, and was not detected in purified $\mathrm{CD} 8 \alpha^{+}$intraepithelial lymphocytes preparations (Figure 1e, and data not shown). Together these results show that colonic and small intestinal epithelial cells express and secrete large amounts of CCL6 into the gut lumen.
Finally, as homeostatic expression of the antibacterial lectin RegIII $\gamma$ in the intestine is dependent on TOLL-like receptor signaling, ${ }^{29}$ we determined whether constitutive CCL6 expression in the ileum was TOLL-like receptor dependent. Although RegIII mRNA levels were reduced 10-fold in the ileum of MyD88 $8^{-/-}$ mice CCL6 transcript levels were unchanged in $\mathrm{MyD}^{-1-}$ and in the Trif ${ }^{-1-}$ mice (data not shown). Thus, TOLL-like receptor signaling is not required to drive the high constitutive expression of CCL6 in intestinal epithelial cells.

\section{Regulation of CCL6 expression}

To examine whether epithelial-derived CCL6 is enhanced by cytokines, CCL6 mRNA expression was analyzed in the murine small intestinal cell line, $\mathrm{mIC}_{\mathrm{c12}},{ }^{30}$ after stimulation with $\mathrm{TNF} \alpha$, IFN $\gamma$, or IL- 4 for $24 \mathrm{~h}$. All three cytokines induced a significant increase in CCL6 transcription (Figure 2a). To determine whether CCL6 expression is enhanced in response to inflammatory stimuli in vivo, mice were injected with $100 \mu \mathrm{g}$ LPS i.p. and CCL6 mRNA expression in the small intestine was assessed over time (Figure 2b). ${ }^{13}$ CCL6 mRNA levels increased significantly $12 \mathrm{~h}$ after LPS injection and returned to steady-state levels by $30 \mathrm{~h}$ (Figure 2b). Hence, intestinal CCL6 mRNA levels are further enhanced in response to inflammatory stimuli.

\section{Recombinant CCL6 and intestinal-derived CCL6 bind to the surface of $E$. coli and a subset of intestinal bacteria}

Given the high constitutive expression of CCL6 by crypt epithelial cells and its presence in the gut lumen, we hypothesized that CCL6 may act as an antimicrobial peptide. In initial studies, we determined whether CCL6 could bind to bacterial surfaces. E. coli were incubated with recombinant CCL6, washed, stained with anti-CCL6 antibody, and analyzed by flow cytometry. Anti-CCL6 antibody bound to E. coli that had been incubated with CCL6, but not to E. coli incubated with control peptide (CCL20), or no peptide (Figure 3a and b, data not shown). Recombinant CCL6 also bound to a subset of freshly isolated intestinal bacteria (Figure $3 \mathbf{a}$ and $\mathbf{b}$ ). To determine whether intestinal epithelial-derived CCL6 could bind to E. coli and intestinal bacteria, antimicrobial extracts were prepared from mouse small intestine and colon as previously described ${ }^{31}$ and incubated with the bacteria. Under these conditions $E$. coli and a subset of intestinal bacteria stained with anti-CCL6 but not a control isotype antibody (Figure 3c). The final concentration of CCL6 present in this assay was 3-5 nM (data not shown) indicating that low concentrations of intestinal-derived CCL6 were sufficient to detect binding to the bacterial surface.

\section{CCL6 displays antibacterial activity at concentrations approximating those found at the intestinal surface} AMPs fulfill a number of biophysical and biochemical properties. ${ }^{32}$ AMPs in general and antimicrobial chemokines in particular have usually an overall positive charge ( $\mathrm{pI}>7)$ and display an amphipathic structure with a positively charged C-terminal $\alpha$-helix. ${ }^{32}$ CCL6 has a pI value of 9.21 and a positively charged C terminus, similar to the antimicrobial chemokine CCL20 ${ }^{18}$ 
a

\begin{tabular}{|l|l|r|r|r|r|}
\hline \multirow{2}{*}{ Chemokine } & \multirow{2}{*}{ Synonyme } & \multicolumn{3}{|c|}{ Relative fluorescence intensity } \\
\cline { 3 - 6 } & & \multicolumn{2}{|c|}{ Small intestine } & \multicolumn{2}{|c|}{ Colon } \\
\cline { 3 - 6 } & & Wildtype & Germfree & Wildtype & Germfree \\
\hline \multirow{2}{*}{ CCL5 } & RANTES & 45 & $\mathrm{~A}$ & $\mathrm{~A}$ & 13 \\
CCL6 & C-10 & 1182 & 695 & 1188 & 864 \\
CCL9 & MRP-2 & 94 & 159 & 87 & 101 \\
CCL25 & TECK & 3574 & 2919 & 48 & 102 \\
CCL27 & CTACK & 23 & 11 & 23 & 20 \\
CXCL11 & I-TAC & 84 & 104 & $\mathrm{~A}$ & $\mathrm{~A}$ \\
CXCL13 & BLC & $\mathrm{A}$ & $\mathrm{A}$ & 40 & $\mathrm{~A}$ \\
CXCL14 & BRAK & 49 & 51 & 56 & 24 \\
CXCL16 & B6 & 79 & 43 & $\mathrm{~A}$ & 16 \\
CX3CL1 & Fractalkine & 290 & 195 & 125 & 184 \\
\hline
\end{tabular}

b

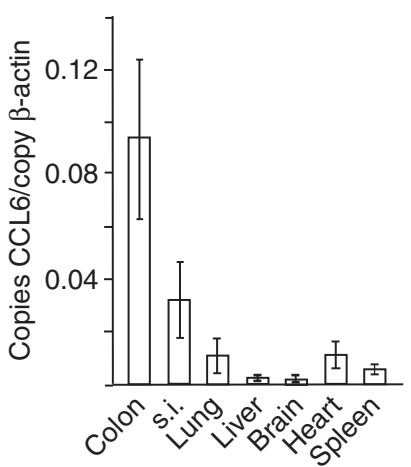

C
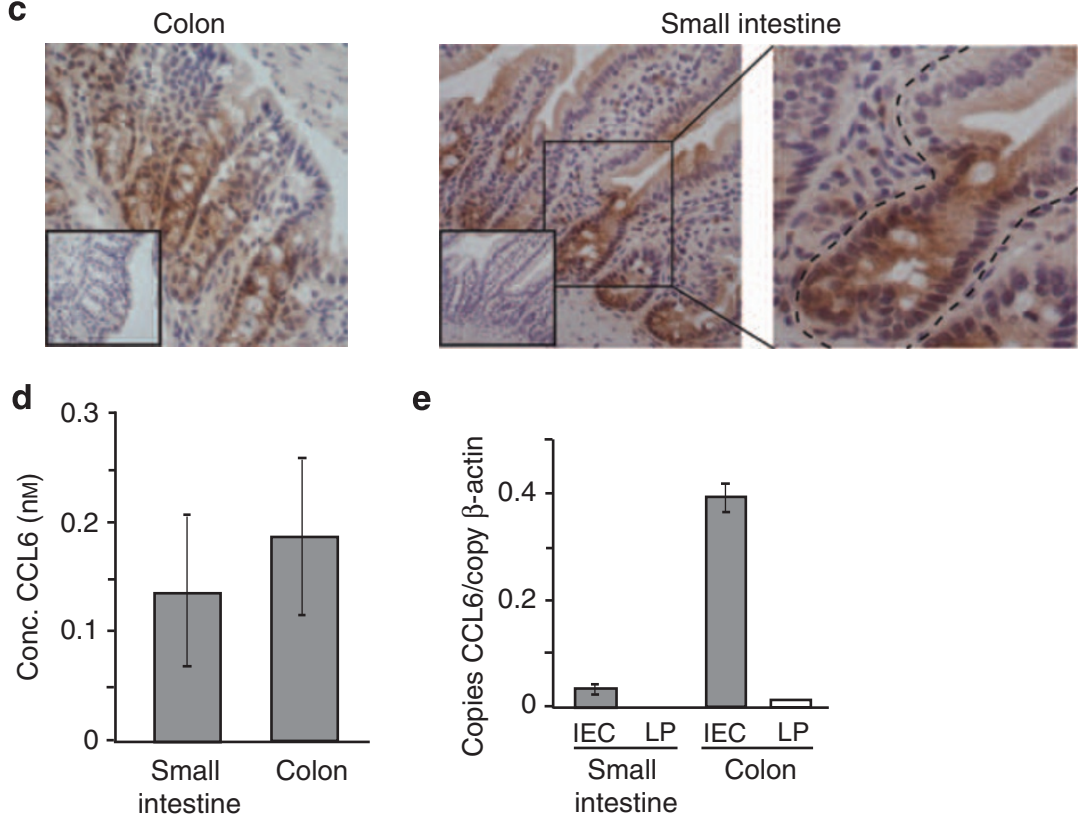

e

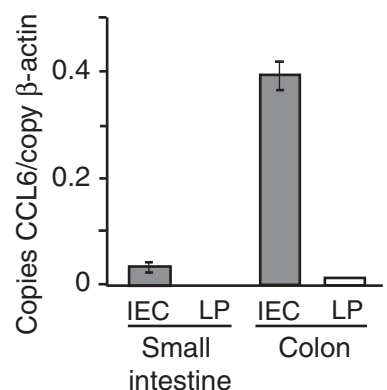

Figure 1 CCL6 is highly expressed by murine intestinal epithelial cells. (a) Chemokine mRNA levels from microarray analysis of laser capture microscopy isolated intestinal epithelial cells from WT and germ-free mice. All chemokines identified as present are listed. The absolute fluorescence intensities are given. $(A=$ absent call). (b) CCL6 mRNA levels in murine tissues were determined by qRT-PCR. Mean ( \pm s.e.m.) of 3-6 mice. s.i., small intestine. (c) Immunohistochemical analysis for CCL6 in murine intestine. Sections were stained with anti-CCL6 or isotype antibody (inlays). Representative stainings from three mice. Prominent CCL6 staining at the luminal site of the epithelium (enlarged micrograph). (d) CCL6 levels in intestinal washes (see Methods) as determined by ELISA. Mean ( \pm s.e.m.) of 4-6 mice. (e) Epithelial cells are the major source of CCL6 mRNA in the murine intestine. CCL6 mRNA levels were determined in epithelial and lamina propria preparations by qRT-PCR. Mean ( \pm s.e.m.) of three mice. IEC, intestinal epithelial cells; LP, lamina propria.

and well above that of CCL3, a chemokine that does not display antibacterial activity ${ }^{18}$ (Figure 4a).

To determine whether CCL6 has antibacterial activity, log phase E. coli were incubated together with recombinant CCL6 (Figure 4b). In viable count assays recombinant CCL6 killed E. coli with a half maximum killing concentration of approximately $200 \mathrm{nM}$ (Figure $4 \mathbf{b}$ ). N-terminal truncation enhances the chemotactic activity of CCL6, ${ }^{33}$ however truncated CCL6 displayed similar antibacterial activity (data not shown). In contrast, CCL6 $(1 \mu \mathrm{M})$ bound poorly to and showed limited ability to kill the pathogenic fungi Candida albicans (Supplementary Figure $2 \mathrm{~A}-\mathrm{C}$ online).

To examine whether CCL6 displays antibacterial activity to potential enteric pathogens we tested its activity on 3 wild-type, virulent Salmonella strains: S. typhimurium SL1344, 14028 and S. enterica Dublin lane. ${ }^{34-36}$ CCL6 had similar antibacterial activity to $S$. enterica Dublin lane as toward $E$. coli but failed to kill the two S. typhimurium isolates 14028 and SL1344 (Figure 4c).

Finally, to compare the antibacterial activity of CCL6 with other established AMPs we incubated $E$. coli with increasing concentrations of CCL6, CCL20, or the human $\beta$-defensin 3 (hBD-3) in a 96-well plate assay. ${ }^{37}$ The minimal inhibitory concentrations (MIC) required to prevent any bacterial growth were determined to be $750 \mathrm{nM}$ for CCL6, $700 \mathrm{nM}$ for human $\beta$-defensin 3 and $608 \mathrm{nM}$ for CCL20 (Figure 4d). Thus, the observed minimal inhibitory concentrations for CCL6 in this assay was similar to that of two established AMPs.

The antibacterial assays described above fail to differentiate between a bacteriostatic or bacteriocidal activity of CCL6. Thus, to determine whether CCL6 displays bacteriocidal activity, and whether it can kill bacteria present within the normal intestinal microflora, E. coli or freshly isolated intestinal bacteria were 

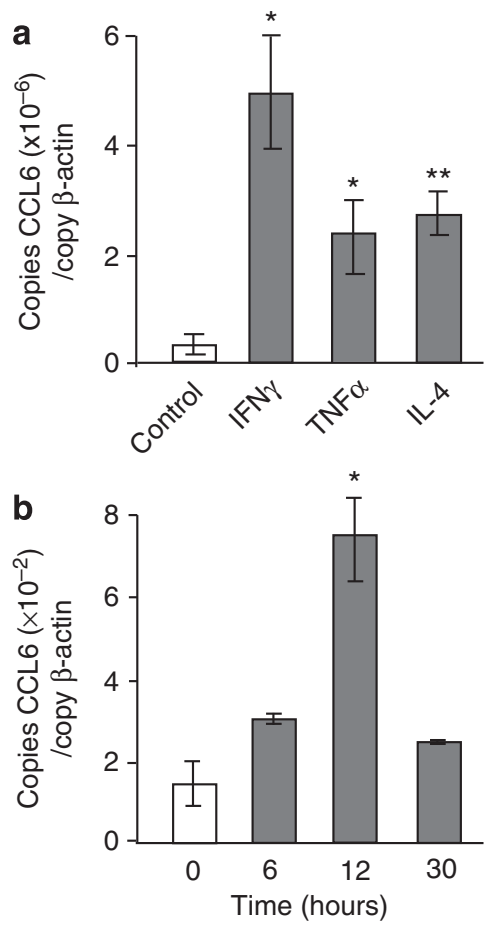

Figure 2 CCL6 expression is enhanced in the intestine by inflammatory mediators. (a) CCL6 mRNA expression by the murine small intestinal cell line $\mathrm{mIC}_{\mathrm{c} 12}$ is enhanced after stimulation with cytokines. TNF $\alpha$ $\left(30 \mathrm{ng} \mathrm{ml}^{-1}\right)$, IFN $\gamma\left(300 \mathrm{ng} \mathrm{ml}^{-1}\right)$, and IL-4 $\left(20 \mathrm{ng} \mathrm{ml}^{-1}\right)$ were added $24 \mathrm{~h}$ before cell harvest. CCL6 mRNA levels were assessed by qRT-PCR. Mean ( \pm s.e.m.) of three separate experiments. (b) Small intestinal CCL6 mRNA expression is enhanced by inflammatory stimuli. Mice were injected with $100 \mu \mathrm{g}$ LPS i.p. and killed at the indicated time points. Mean ( \pm s.e.m.) of $3-5$ mice/time point. ${ }^{\star} P \leqslant 0.05$ and ${ }^{\star \star} P<0.01$. TNF $\alpha$, tumor necrosis factor- $\alpha$; IFN $\gamma$, interferon- $\gamma$.

incubated with CCL6 (50 nM-1 $\mu \mathrm{M})$ and depolarization of bacterial membranes was assessed by staining with $\mathrm{DiBAC}_{4}$ as described. ${ }^{31}$ CCL6 dose dependently induced membrane depolarization of E. coli and intestinal bacteria (Figure 4e). Thus, CCL6 can bind to the surfaces of certain bacteria and displays bacteriocidal activity.

To determine whether physiologically relevant levels of CCL6 required for antibacterial activity are present at the small intestinal and colonic mucosal surface, we determined the CCL6 concentration in mucosal scrapings (see Methods) by ELISA (Figure 4f). CCL6 levels were lower than the observed halfmaximal killing dose observed in viable count assays, and in the range necessary to induce bacterial membrane depolarization. We would nevertheless predict that concentrations of CCL6 specifically in crypt regions, in which the majority of CCL6 is located (Figure 1c), are considerably higher and thus that CCL6 has a significant role as an antibacterial agent in this region.

\section{The human homologs of mouse CCL6, CCL14, and CCL15, are constitutively expressed in the human intestine and display antibacterial activity}

The closest human homologs to CCL6, based on protein sequence alignments and as previously suggested, ${ }^{38-40}$ are CCL14 and CCL15 (Supplementary Figure 3A online).
Similar to CCL6, CCL14 and CCL15 have a positive net-charge and positively charged C terminus (Supplementary Figure 3B online). qRT-PCR on micro-dissected small intestinal epithelial cells showed high constitutive expression of both CCL14 and CCL15 mRNA (Figure 5a). Importantly, both CCL14 and CCL15 displayed similar antibacterial activity as CCL6 in viable count assays (Figure 5b). Thus, CCL14 and CCL15 show a high degree of sequence similarity to mouse CCL6, are constitutively expressed by intestinal epithelial cells and share its antibacterial properties.

\section{CCL14 and CCL15 expression is enhanced in inflammatory bowel disease}

To determine whether CCL14 and CCL15 transcription is enhanced under inflammatory conditions, the human colonic epithelial cell line HT-29 was stimulated with IFN $\gamma, \mathrm{TNF} \alpha$, or IL-4 for $24 \mathrm{~h}$ and CCL14 and CCL15 mRNA expression assessed by qRT-PCR (Supplementary Figure 3 online). IFN $\gamma$ and TNF $\alpha$ significantly enhanced CCL14 mRNA expression, whereas IL-4 had no significant effect. In contrast, only IFN $\gamma$ significantly enhanced CCL15 transcription. Finally, given the antibacterial activity of CCL14 and CCL15 and their constitutive expression in the small intestine and colon, we determined whether levels of intestinal CCL14 and CCL15 were altered in patients with IBD. IBD is classified into two major diseases; ulcerative colitis affecting primarily the colon and Crohn's disease that may affect the entire gut including both the colon and ileum. Therefore, colonic biopsies derived from control and IBD patients were analyzed for their expression of CCL14 and CCL15 by quantitative real-time PCR. Both, CCL14 and CCL15 mRNA transcripts were highly expressed in the colon and levels were significantly enhanced in Crohn's and ulcerative colitis patients (Figure 5c). Together, these results suggest that CCL14 and CCL15 contribute as AMPs to the innate mucosal immune defense in the steady state and during chronic intestinal inflammation.

\section{DISCUSSION}

Although chemokines have important roles in regulating immune cell localization to and function within the intestinal mucosa, $12-16,26,41,42$ their role as antibacterial peptides in the intestinal mucosa remains unclear. To fulfill such a function, the chemokine should not only display antimicrobial activity but also be present in the mucosa at relevant antibacterial concentrations.

CCL6 has previously been reported to be expressed by cells of the monocyte/macrophage lineage, eosinophils, and keratinocytes ${ }^{43-45}$ and at high levels in lung tissue ${ }^{27,28}$ with minor expression in testis, heart, and ovary. ${ }^{28}$ Here, we made the unexpected discovery that the highest constitutive expression of CCL6 is observed in the intestine. Even more striking is our finding that epithelial cells are the major source of intestinal CCL6 and that CCL6 is one of the most abundant chemokines expressed by small intestinal and the most abundant chemokine expressed by colonic epithelial cells. Given the antimicrobial activity described for several chemokines, we hypothesized that CCL6 may function in the crypt region as an antibacterial peptide. In agreement with this hypothesis, CCL6 contains structural motifs associated 


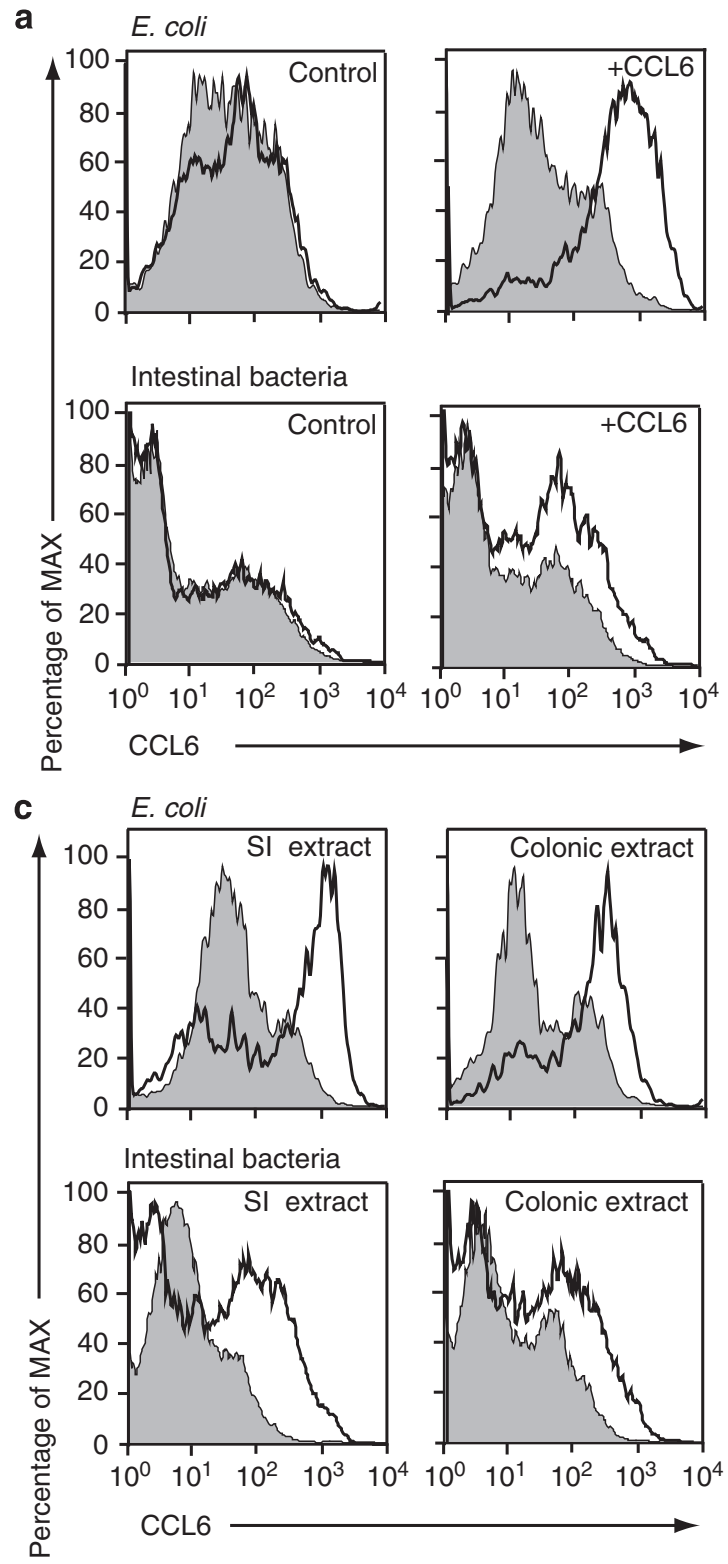

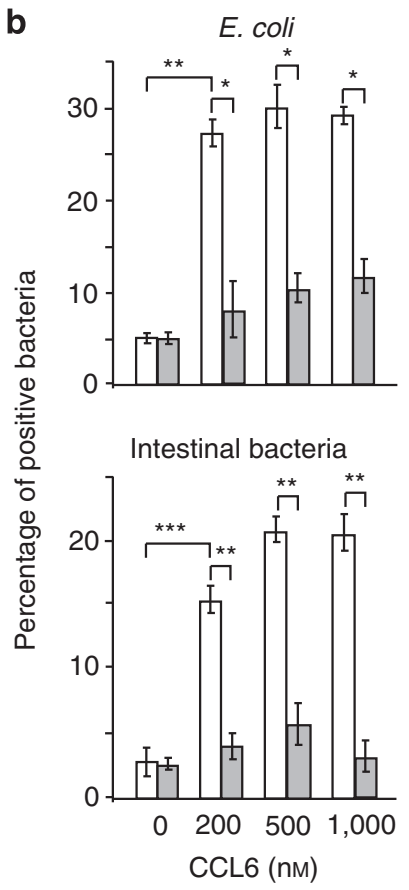

Anti-CCL6 Isotype

Figure 3 CCL6 binds to the surface of bacteria. (a and $\mathbf{b})$ E. coli or freshly isolated intestinal bacteria were incubated with recombinant CCL6 at the indicated concentrations, stained with anti-CCL6 or control antibody and analyzed by flow cytometry. (a) Representative FACS plots. Anti-CCL6 (blank) or isotype control antibody (filled) (b). Mean ( \pm s.e.m.) results of three independent experiments. ${ }^{*} P<0.05 ;{ }^{* \star} P<0.01 ;{ }^{* \star *} P<0.001$. (c) CCL6 present in tissue extracts binds to $E$. coli and intestinal bacteria. Bacteria were incubated with small intestinal or colonic epithelial extracts for $2 \mathrm{~h}$ and stained with anti-CCL6 or isotype antibody. Data are representative FACS plots (One representative experiment of 3-4 performed). E.coli, Escherichia coli.

with antibacterial chemokines ${ }^{18,19}$ and both recombinant- and tissue-derived CCL6 bound to and displayed antibacterial activity towards E. coli, Salmonella enterica and a subset of the normal intestinal bacterial flora. As the bacterial flora is composed of a wide variety of bacterial species and genera, ${ }^{46}$ these results suggest that the effect of CCL6 might be restricted to defined bacterial subgroups. Indeed, although CCL6 displayed antibacterial activity to Salmonella enterica Dublin Lane it showed no activity against two Salmonella typhimurium strains. Such selective activity has been observed for other AMPs. ${ }^{29}$ For example, the intestinal killing of Listeria monocytogenes completely depends on the expression of the antibacterial lectin RegIIIr. ${ }^{29}$ It is interesting to note that CCL6 failed to bind and kill the eukaryotic pathogenic fungus Candida albicans suggesting a correlation between binding and killing. Nevertheless more work will be required to determine whether the antibacterial and binding activity of CCL6 are directly linked.

To our knowledge, AMP concentrations at the intestinal surface have not been previously assessed; however, a few studies have determined AMP levels at other mucosal sites. Human hBD-3 reaches concentrations of $50 \mathrm{nM}$ in saliva samples, ${ }^{47}$ whereas levels of hBD-1 and hBD-2 in vaginal fluid of healthy volunteers reached 10 and $20 \mathrm{nM}$, respectively. ${ }^{48}$ In vitro half-maximal antibacterial activity has been reported at $500-2,000 \mathrm{nM}$ for hBD- $3^{49}$ and at $1 \mu \mathrm{M}$ and $120 \mathrm{nM}$ for hBD- 1 or hBD-2, respectively. ${ }^{50}$ The antibacterial chemokines, CXCL9 


\begin{tabular}{|l|l|l|}
\hline Chemokine & $\mathrm{pl}$ & C-term. $\alpha$-helix \\
\hline CCL6 & 9.5 & DRRVQRCLSTLK \\
CCL20 & 9.21 & PKQTWVKYIVRLL SKKVK \\
CCL3 & 4.77 & SEEWVQ \\
\hline
\end{tabular}

b

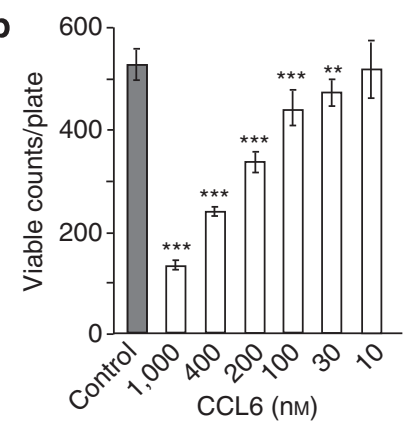

C

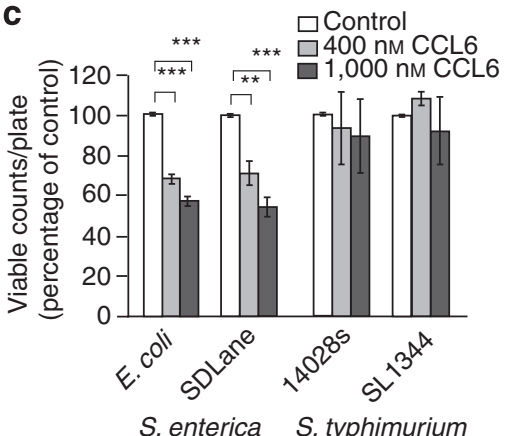

S. enterica S. typhimurium

d

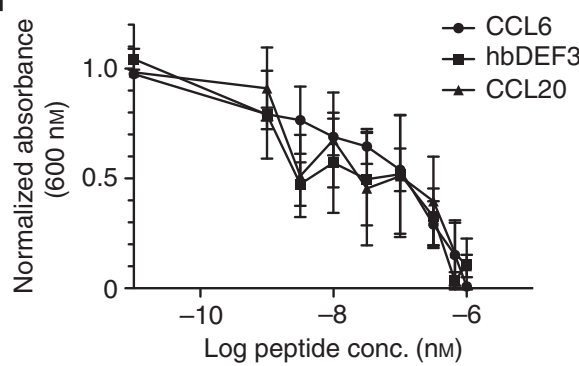

Figure 4 CCL6 displays antibacterial activity. (a) CCL6 share structural and physicochemical properties similar with the antibacterial chemokine CCL20 in contrast to CCL3 displaying no antibacterial activity. (b) CCL6 displays anti-bacterial activity. Log phase E. coli were incubated with CCL6 and the bacterial viability is assessed in viable count assay. Results are from one representative experiment out of four performed. Mean ( \pm s.e.m.) for $n=4-8$ agar plates (2-3 agar plate/dilution; three different dilutions assayed). ${ }^{* \star} P<0.01$ and ${ }^{* * *} P<0.001$. (c) CCL6 displays antibacterial activity against Salmonella enterica. Log phase bacteria were incubated with CCL6 and the viability of bacteria assessed in a viable count assay. Mean ( \pm s.e.m.) from three experiments. (black bars, 1,000 nM CCL6; gray bars, $400 \mathrm{nM} \mathrm{CCL6,} \mathrm{open} \mathrm{bars,} \mathrm{control)}$ ${ }^{\star \star} P<0.01$ and ${ }^{* \star *} P<0.001$. (d) Comparison of the antibacterial activity of CCL6 with established AMPs. CCL6, CCL20, or human $\beta$-defensin 3 in the minimal inhibitory concentrations assay. Results shown represent an average of three to four experiments performed ( \pm s.e.m.). (e) CCL6 induces membrane depolarization in $E$. coli and gut bacteria. Bacteria were incubated with CCL6 for $2 \mathrm{~h}$ and depolarization was monitored by $\mathrm{DiBAC}_{4}$ staining. Mean ( \pm s.e.m.) of 3-5 experiments. Results are percent $\mathrm{DiBAC}_{4}$-positive (depolarized) bacteria with control (no CCL6) subtracted. ${ }^{\star \star} P<0.01$ and ${ }^{\star * *} P<0.001$. (f) CCL6 protein concentration in intestinal scrapings. CCL6 concentrations were determined by ELISA. Mean ( \pm s.e.m.) of four mice. S.I., small intestine. E.coli, Escherichia coli.
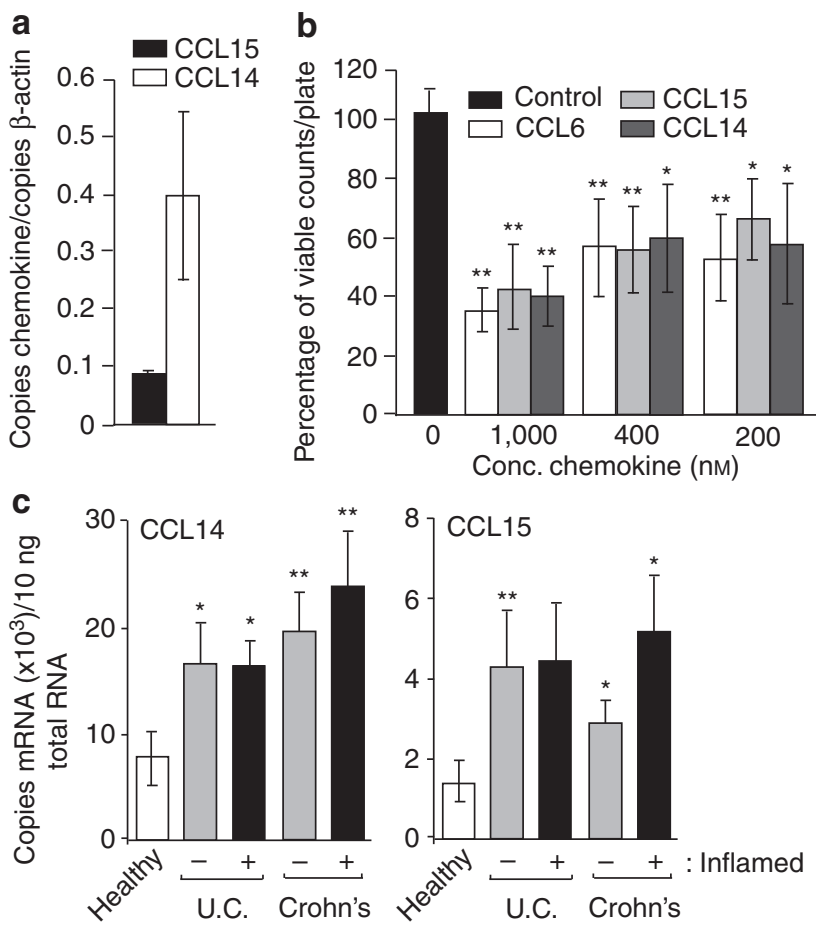

Figure 5 CCL14 and CCL15 are expressed by human intestinal epithelial cells, display antibacterial activity, and are enhanced in IBD. (a) Epithelial cells are the source of CCL14 and CCL15 mRNA. Small intestinal epithelial cells were isolated from tissue sections and CCL14 and CCL15 mRNA levels were determined by qRT-PCR. Mean ( \pm s.e.m.) of two experiments (samples from two patients). (b) CCL14 and CCL15 kill bacteria. E. coli were incubated with the indicated chemokines or control for $2 \mathrm{~h}$ and antibacterial activity was assessed in viable count assays. Mean ( \pm s.e.m.) of three experiments (4-6 agar plates/data point/experiment). ${ }^{*} P \leqslant 0.05,{ }^{* \star} P<0.01$. (c) CCL14 and CCL15 mRNA expression is upregulated in the colon in ulcerative colitis (u.c.) and Crohn's disease (Crohn's). CCL14 and CCL15 mRNA levels in human colonic biopsies were assessed by qRT-PCR. Mean ( \pm s.e.m.) from 10 to 27 biopsies/group. ${ }^{\star} P \leqslant 0.05,{ }^{\star \star} P<0.01$. E.coli, Escherichia coli.

and CXCL10 reach concentrations of $10-50 \mathrm{nM}$ and 1-7 nM, respectively, in patients tonsil fluid during streptococcal pharyngitis $^{21}$ and the half-maximal antimicrobial activity observed was $30 \mathrm{nM}$ for CXCL9 and $200 \mathrm{nM}$ for CXCL10. ${ }^{21}$ Thus, the reported levels of acknowledged AMPs at mucosal tissue are lower or within the range required to induce half-maximal killing in vitro. In this study CCL6 concentrations in intestinal scrapings were approximately $30-50 \mathrm{nM}$ (Figure 4f), close to concentrations that induce bacterial membrane depolarization, and slightly lower than the concentration required for half-maximal killing in viable count assays. As CCL6 measurements represented average levels from the entire intestinal surface it is reasonable to assume that CCL6 reaches considerably higher levels in the epithelial crypt region where the majority of CCL6 protein localized. Thus our findings suggest that CCL6 represents an important epithelialderived antimicrobial chemokine in intestinal crypts.

CCL14 and CCL15 are human homologs to CCL6 displaying 36 and 53\% amino acid identity to CCL6, respectively. Northern blot analysis has shown constitutive CCL14 and CCL15 expression in the human small intestine and colon. ${ }^{39,51}$ Despite this, a function for CCL14 and CCL15 and the cellular source of these 
chemokines in the intestinal mucosa has yet to be described. Here, using qRT-PCR on laser-captured intestinal samples we show that epithelial cells are a major source of CCL14 and CCL15 mRNA and that the levels of CCL14 are approximately fivefold higher than CCL15. Both chemokines displayed biochemical properties associated with antibacterial chemokines ${ }^{7}$ and both displayed similar antibacterial activity to CCL6. Thus CCL14 and CCL15 are both structural and functional homologs to CCL6 and represent antibacterial chemokines of the human intestinal mucosa.

AMP expression is reportedly enhanced in the colon of ulcerative colitis patients ${ }^{52,53}$ and intestinal extracts from these patients display increased antibacterial activity ${ }^{54}$ In colons affected by Crohn's disease several AMPs are unchanged at the mRNA levels and the total antibacterial activity in $\mathrm{CD}$ colon biopsies has been reported to be reduced ${ }^{54}$ Here, we found significantly enhanced expression of CCL14 and CCL15 in both inflamed and non-inflamed areas of the colon in ulcerative colitis and Crohn's patients. Our finding that IFN $\gamma$ and TNF $\alpha$ enhanced epithelial production of CCL14 and CCL15, respectively, suggests that these cytokines, potentially produced by infiltrating leukocytes, are in part responsible for the upregulation of these chemokines in IBD. This enhanced expression of CCL14 and CCL15 also suggest that both chemokines likely contribute to enhancing the antibacterial activity of the intestinal mucosa in IBD.

Although our current study focuses on the role of CCL6, CCL14, and CCL15 as AMPs, these chemokines also act as leukocyte chemoattractants, primarily acting on cells of the monocyte/macrophage lineage. . $^{28,38,39,51,55,56}$ It thus seems possible that CCL6, CCL14, and CCL15 have additional roles in regulating immune cell recruitment and/or function in the intestine. Consistent with this possibility a subset of murine $\mathrm{CD} 11 \mathrm{~b}^{+}$, intestinal dendritic cells express CCR $1,{ }^{57}$ a putative CCL6 receptor. ${ }^{33}$ Nevertheless, in preliminary experiments we have found that $\mathrm{CCR} 1^{-/-}$bone marrow is equally efficient at generating $\mathrm{MHCII}^{+} \mathrm{CD} 11 \mathrm{c}^{+}$intestinal dendritic cell subsets in competitive bone marrow chimeras (authors unpublished observation) indicating that CCR1/CCL6 are not required for intestinal dendritic cell recruitment in the steady state. Further studies determining the role of CCR1 in regulating myeloid/dendritic cell recruitment in the setting of intestinal inflammation are clearly warranted.

In conclusion, we demonstrate that the chemokine CCL6, and its human homologs CCL14 and CCL15 are highly and constitutively expressed in intestinal epithelial cells, have structural properties of AMPs and display antibacterial activity. Our results point to a novel role for a subgroup of intestinal epithelialderived chemokines as AMPs that have a potentially important role in maintaining crypt integrity and intestinal homeostasis.

\section{METHODS}

\section{Mice}

C57BL/6 mice were housed in the Bio Medical Center animal facility, Lund University, Sweden. All mice were kept with free access to standard rodent chow diet and drinking water and were regularly checked by the animal house operational personal. Germ-free (12 mice) and conventionally reared C57BL/6 control mice were obtained from the Division of Molecular Pathology, Karolinska Institute. All animal studies were approved by the Malmö/Lund Animal Ethics Committee.

\section{Laser capture microscopy and microarray analysis}

Laser capture microscopy was performed as described. ${ }^{23}$ Total RNA was extracted from 16,000 catapulted cells (pooled from four individual mice) using RNA microprep kit (Stratagene, Stockholm, Sweden). Total RNA (40 ng) was expanded by two rounds of in vitro cRNA amplification and affymetrix genechip Moe403 were probed at the Swegene Center Lund, Sweden. Array analysis was performed using dCHIP2004.

\section{Isolation of murine intestinal cell populations}

Epithelial cells and intraepithelial lymphocytes were isolated from the murine intestine as described. ${ }^{15,23}$

\section{Quantitative real-time PCR}

qRT-PCR was performed as described. ${ }^{23}$ Plasmids containing cloned CCL14 and 15 or PCR amplified CCL6 were used as internal standards. Product identity was confirmed by sequencing. qRT-PCR of laser-captured samples was performed as described. ${ }^{58}$ Primer sequences are specified below. CCL6 forward: 5' ATGAGAAACTCCAAGACTGCC 3';

CCL6 reverse: 5' TTATTGGAGGGTTATAGCGACG 3';

CCL15 forward 5' TGGATCCCAGGCCCAGTTCATA 3';

CCL15 reverse 5' GATGCAGAGACAGAGTTAATGAT 3';

CCL15 inner forward 5' TGACACCTGGCTTGGAGCACTC 3';

CCL15 inner reverse 5'CCCCTTCTTGGTGAGGAATATG 3';

CCL14 forward 5' ATCTCCGTGGCTGCCATTCCCTT 3';

CCL14 reverse 5' AGGGACCAAGACTGAATCCTCC 3';

CCL14 inner forward 5' GGAATGGCCCCTTTTGGTGAT 3';

CCL14 inner reverse 5'CCACTTGTCACTGGGGTTGGTACA 3';

$\beta$-actin forward 5' CCGGGACCTGACAGACTA 3';

$\beta$-actin reverse 5' AGAGCCTCAGGGCATCGGAAC 3';

$\beta$-actin inner forward 5' CTCATGAAGATCCTGACCGAGC 3';

$\beta$-actin inner reverse 5' GTTTCATGGATGCCACAGGAT 3'

RegIII $\gamma$ fw $5^{\prime}$ GTATAACCATCACCATCATGTCC $3^{\prime}$ and

RegIII $\gamma$ rev 5' GAAGCTTCAGCGCCACTGAGC 3'.

\section{Immunohistochemistry.}

Tissue sections $(7 \mu \mathrm{m})$ were fixed in $4 \%$ paraformaldehyde. Endogenous peroxidase activity was blocked $\left(10 \mathrm{~min}\right.$ in $\left.1 \% \mathrm{H}_{2} \mathrm{O}_{2}\right)$ and sections were incubated with polyclonal goat anti-mouse CCL6 (R and D Systems, Abingdon, United Kingdom) or goat IgG $\left(3 \mu \mathrm{g} \mathrm{ml}^{-1}\right) \mathrm{O} / \mathrm{N}$ at $4^{\circ} \mathrm{C}$ overnight. Washed sections were incubated with secondary rabbit anti-goat horseradish peroxidase-labeled antibody (1:1,000 P0449, Dako, Glostrup, Denmark) for $2 \mathrm{~h}$ at RT. Immunostaining was visualized using DAB peroxidase-chromogen reaction (Sigma-Aldrich, Stockholm, Sweden). Sections were counterstained with Mayer's hematoxylin $(5 \mathrm{~min})$, dehydrated, mounted using Mountex (HistoLab Products, Gothenburg, Sweden) and visualized with a Zeiss Axiovert 200/M microscope using Volocity software (Improvision, Perkin-Elmer, Upplands Väsby, Sweden).

\section{Chemokine induction assays}

In vivo: C57BL/6 mice were injected with LPS (100 $\mu$ g, serotype O55:B5; Sigma-Aldrich) or PBS, killed at the time point indicated and ileum was isolated for qRT-PCR analysis. Epithelial cell line stimulation; $\mathrm{mIC}_{\mathrm{cl2}} \mathrm{cells}^{30}$ were grown to confluence and stimulated with the indicated cytokines. ${ }^{23}$

HT-29 cells (150,000 cells) were seeded into 24 -well plates (NUNC) and grown for 3 days in DMEM containing $10 \% \mathrm{FBS}$ at $37^{\circ} \mathrm{C}$ and $5 \% \mathrm{CO}_{2}$. Thereafter, medium was replaced with DMEM containing 2\% FBS. One day later cytokines ( $\mathrm{R}$ and $\mathrm{D}$ Systems) were added to confluent cultures and cells were harvested after a $24 \mathrm{~h}$ incubation for qRT-PCR analysis.

Determination of intestinal CCL6 concentrations. Intestinal washes (Figure 1d): Mouse intestinal content was collected by flushing through with $1 \mathrm{ml}$ cold PBS. The flush through was vortexed, centrifuged at 14,000 r.p.m. for $5 \mathrm{~min}$ and CCL6 concentrations were determined by ELISA. In intestinal scrapings (Figure 4e): Mouse intestine was flushed through with $10 \mathrm{ml}$ PBS to remove the luminal content. The intestine was opened longitudinally and the mucosal surface, including mucus, gently scraped 
with a scalpel blade, as described. ${ }^{59}$ The scraped volume was diluted 1:1 in PBS, vortexed, and CCL6 concentrations determined by ELISA.

CCL6 ELISA. Maxisorb ELISA plates (NUNC, Fisher Scintific GTF, Sweden) were coated with polyclonal goat anti-mouse CCL6 antibody $\left(0.2 \mathrm{mg} \mathrm{ml}^{-1}\right) \mathrm{O} / \mathrm{N}$. After washing and blocking, standards and samples were added to the plate and incubated for $2 \mathrm{~h}$ at RT. CCL6 was detected with monoclonal rat-anti-mouse CCL6 (Clone 262016, R and D Sytems) followed by an anti-rat horse-radish-peroxidase coupled secondary antibody (212-036-168, Jackson Laboratories, Newmarket, Suffolk, England) and visualized using ELISA detection reagent ( $\mathrm{R}$ and $\mathrm{D}$ Systems).

\section{Isolation of antimicrobial extracts}

Isolation of intestinal antimicrobial peptides was performed as described. ${ }^{54}$ Briefly, epithelial cells were isolated as described ${ }^{15,23}$ and extracted using $5 \%$ acetic acid, supplemented with Complete-mini protease inhibitor cocktail (Roche Applied Science, Bromma, Sweden). After extraction, lysates were centrifuged for $15 \mathrm{~min}$ at $560 \times g$, and the supernatant was recovered. The supernatant was dried under vacuum at room temperature and the resulting pellet resolved in $4 \mathrm{ml} 0.01 \%$ acetic acid. Cationic peptides were further purified by ion exchange chromatography using Macroprep CM matrix (Biorad, Sundbyberg, Sweden). Antimicrobial peptides were subsequently eluted with $2 \mathrm{ml} 5 \%$ acetic acid, dried under vacuum and resolved in $200 \mu \mathrm{l} 0.01 \%$ acetic acid in Tris- $\mathrm{HCl}(25 \mathrm{mM}$, $\mathrm{pH}$ 7.2). Finally, the extract was dialyzed using Spectrapor dialysis tubing with a pore size of $3.5 \mathrm{kDa}$ (Spectrumlabs, Breda, Netherlands).

\section{Purification of intestinal bacteria from the murine intestine}

Mouse cecum and colon was removed and luminal contents flushed out with $20 \mathrm{ml}$ PBS. After vortexing, $1 \mathrm{ml}$ of intestinal contents was subjected to repetitive centrifugation in $20 \mathrm{mM}$ Hepes- $\mathrm{HCl} \mathrm{pH} 7.2$ at $240 \times g$ for $3 \mathrm{~min}$ (to remove cell debris and food aggregates) and thereafter at $20,800 \times g$ for $3 \mathrm{~min}$. This procedure was repeated 6-7 times. Purity of the bacterial preparation was confirmed by ocular inspection under a light microscope.

\section{Staining for surface bound CCL6}

E. coli XL10 Gold (Stratagene), intestinal bacteria, or Candida albicans (ATCC 90028) $\left(\sim 2.5-5 \times 10^{5}\right.$ microbes) were incubated together with CCL6 (R and D Systems), control peptide (CCL20) or alone in $20 \mathrm{mM}$ Hepes- $\mathrm{HCl} \mathrm{pH} 7.2$ at $37^{\circ} \mathrm{C}$ for $2 \mathrm{~h}$. Samples were blocked with $10 \%$ normal mouse serum, and stained with polyclonal goat anti-mouse CCL6 or control goat $\operatorname{IgG}\left(4 \mu \mathrm{g} \mathrm{ml}^{-1}\right)$. After washing, primary antibody binding was detected with biotinylated mouse anti-goat antibody (no. 205-065108, Jackson Laboratories), followed by streptavidin-APC and analyzed on a FACS Calibur (BD Bioscience, Stockholm, Sweden).

\section{Microbial killing assays}

Viable count assay. E. coli XL10 Gold (Stratgene), S. enterica Dublin Lane and S. typhimurium (14028 and SL1344 strains) were grown to an $\mathrm{OD}_{620}=0.4$, washed three times in $20 \mathrm{mM}$ Tris- $\mathrm{HCl}, \mathrm{pH} 7.2$ and bacteria $\left(\sim 5 \times 10^{4}\right)$ were incubated with the indicated amounts of peptide in $50 \mu \mathrm{l} \mathrm{Tris-HCl} \mathrm{buffer} \mathrm{for}$ $2 \mathrm{~h}$. Serial dilutions were prepared, plated on LB Agar plates and bacterial colonies were counted after $\mathrm{O} / \mathrm{N}$ incubation at $37^{\circ} \mathrm{C}$. Minimal inhibitory concentration assay. The 96-well based assay to compare antimicrobial activity of different peptides was performed as previously described. ${ }^{37}$ All peptides used (CCL6, CCL20, and human $\beta$-defensin 3) were purchased from $\mathrm{R}$ and $\mathrm{D}$ Systems. Bacteria were incubated for $2 \mathrm{~h}$ with the indicated peptides in 20 mM Tris- $\mathrm{HCl}$ pH 7.2 followed by incubation in Mueller Hinton Broth. Absorbance at $600 \mathrm{~nm}$ was determined using a 96-well plate ELISA reader (Emax plate reader; Molecular Device, Sunnyvale, CA). Assessment of bacterial viability by flow cytometry. To assess bacterial viability, membrane depolarization was determined using the dye $\mathrm{DiBAC}_{4}$ as described. ${ }^{54}$

\section{Yeast killing assays}

Viable count assay. Candida albicans (ATCC 90028) was grown O/N in YPD medium (Sigma-Aldrich) at $37^{\circ} \mathrm{C}$ on a rotary shaker. To obtain logarithmically dividing Candida, $10 \mathrm{ml}$ of culture were diluted with new YPD medium (1:1) and cultured for another $4 \mathrm{~h}$. Twenty thousand fungi were washed as described above and incubated with the indicated amounts of CCL6 or control peptide for $2 \mathrm{~h}$. Serial dilutions were plated on YPD agar plates and the number of colonies assessed after culture $\mathrm{O} / \mathrm{N}$.

\section{Human colon samples}

Samples for analysis in Figure 5a were obtained from patients undergoing surgery for bladder reconstruction or intestinal cancer in collaboration with T Davidsson and W Månsson (Lund University Hospital) after approval from the Regional Ethics committee in Lund, Sweden and informed patient consent. Colonic biopsies from healthy individuals (controls) and IBD patients (ulcerative colitis and Crohn's disease) in Figure $5 \mathbf{c}$ were obtained and processed at the Robert Bosch Hospital (Stuttgart, Germany). The protocol was approved by the Institutional Review Board. The diagnosis was based on standard criteria using clinical, radiological, endoscopic, and histopathological findings. Exclusion criteria included the diagnoses of indeterminate colitis, concurrent CMV or C. difficile infection. Samples were immediately snap frozen in liquid nitrogen for subsequent analysis.

\section{Statistical analysis}

Significance between groups from all mouse data was assessed by unpaired Student's $t$-test (95\% confidence interval) using the GraphPad's PRISM software. Statistical analyses of qRT-PCR for human samples were performed non-parametrically using the Wilcoxon, and Mann-Whitney $U$-test. Values of $P<0.05$ were considered statistically significant.

SUPPLEMENTARY MATERIAL is linked to the online version of the paper at http://www.nature.com/mi

\section{ACKNOWLEDGMENTS}

We thank Drs H Uronen-Hansson (Immunology Section, Lund University, Sweden), T Davidsson and W Månsson (Department of Urology, Lund University Hospital, Sweden) for human tissue samples, Dr A Vandervalle (Institut National de la Santé et de la Recherche Médicale, Faculté X, Paris, France) for the $\mathrm{mIC}_{\mathrm{c} \mid 2}$ cell line and Dr M J Wick (Department of Microbiology and Immunology, Gothenburg University, Sweden) for providing the Salmonella strains. This work was supported by grants from the Swedish Medical Research Council, the Crafoordska, Richard and Ruth Julins, Österlund, Nanna Svartz and Kocks Foundations, the Royal Physiographic Society in Lund, and the Swedish Foundation for Strategic Research "MicMan" and future research leaders II program;. KK was supported by a SWEGENE Postdoctoral Fellowship.

\section{DISCLOSURE}

The authors declared no conflict of interest.

(C) 2010 Society for Mucosal Immunology

\section{REFERENCES}

1. Bouskra, D. et al. Lymphoid tissue genesis induced by commensals through NOD1 regulates intestinal homeostasis. Nature 456, 507-510 (2008).

2. Artis, D. Epithelial-cell recognition of commensal bacteria and maintenance of immune homeostasis in the gut. Nat. Rev. Immunol. 8, 411-420 (2008).

3. Guarner, F. \& Malagelada, J.R. Gut flora in health and disease. Lancet 361, 512-519 (2003).

4. Salzman, N.H., Ghosh, D., Huttner, K.M., Paterson, Y. \& Bevins, C.L. Protection against enteric salmonellosis in transgenic mice expressing a human intestinal defensin. Nature 422, 522-526 (2003).

5. Zasloff, M. Antimicrobial peptides of multicellular organisms. Nature 415, 389-395 (2002).

6. Cunliffe, R.N. \& Mahida, Y.R. Expression and regulation of antimicrobial peptides in the gastrointestinal tract. J. Leukoc. Biol. 75, 49-58 (2004).

7. Clarke, D.J. \& Campopiano, D.J. Structural and functional studies of defensin-inspired peptides. Biochem. Soc. Trans. 34, 251-256 (2006).

8. Hisamatsu, T. et al. CARD15/NOD2 functions as an antibacterial factor in human intestinal epithelial cells. Gastroenterology 124, 993-1000 (2003).

9. Wehkamp, J. et al. NOD2 (CARD15) mutations in Crohn's disease are associated with diminished mucosal alpha-defensin expression. Gut 53, 1658-1664 (2004). 
10. Kobayashi, K.S. et al. Nod2-dependent regulation of innate and adaptive immunity in the intestinal tract. Science 307, 731-734 (2005).

11. Bonen, D.K. \& Cho, J.H. The genetics of inflammatory bowel disease. Gastroenterology 124, 521-536 (2003).

12. Cook, D.N. et al. CCR6 mediates dendritic cell localization, lymphocyte homeostasis, and immune responses in mucosal tissue. Immunity 12, 495-503 (2000).

13. Tanaka, Y. et al. Selective expression of liver and activation-regulated chemokine (LARC) in intestinal epithelium in mice and humans. Eur. J. Immunol. 29, 633-642 (1999).

14. Kunkel, E.J. et al. Lymphocyte CC chemokine receptor 9 and epithelial thymus-expressed chemokine (TECK) expression distinguish the small intestinal immune compartment: epithelial expression of tissue-specific chemokines as an organizing principle in regional immunity. J. Exp. Med. 192, 761-768 (2000).

15. Svensson, M. et al. CCL25 mediates the localization of recently activated CD8alphabeta(+) lymphocytes to the small-intestinal mucosa. J. Clin. Invest. 110, 1113-1121 (2002).

16. Lazarus, N.H. et al. A common mucosal chemokine (mucosae-associated epithelial chemokine/CCL28) selectively attracts IgA plasmablasts. J. Immunol. 170, 3799-3805 (2003).

17. Cole, A.M. et al. Cutting edge: IFN-inducible ELR- CXC chemokines display defensin-like antimicrobial activity. J. Immunol. 167, 623-627 (2001).

18. Yang, D. et al. Many chemokines including CCL2O/MIP-3alpha display antimicrobial activity. J. Leukoc. Biol. 74, 448-455 (2003).

19. Eliasson, M. \& Egesten, A. Antibacterial chemokines-actors in both innate and adaptive immunity. Contrib. Microbiol. 15, 101-117 (2008).

20. Moser, B., Wolf, M., Walz, A. \& Loetscher, P. Chemokines: multiple levels of leukocyte migration control. Trends Immunol. 25, 75-84 (2004).

21. Egesten, A. et al. The CXC chemokine MIG/CXCL9 is important in innate immunity against Streptococcus pyogenes. J. Infect. Dis. 195, 684-693 (2007).

22. Hieshima, K. et al. CCL28 has dual roles in mucosal immunity as a chemokine with broad-spectrum antimicrobial activity. J. Immunol. 170, 1452-1461 (2003).

23. Ericsson, A., Kotarsky, K., Svensson, M., Sigvardsson, M. \& Agace, W. Functional characterization of the CCL25 promoter in small intestinal epithelial cells suggests a regulatory role for caudal-related homeobox (Cdx) transcription factors. J. Immunol. 176, 3642-3651 (2006).

24. Wurbel, M.A. et al. The chemokine TECK is expressed by thymic and intestinal epithelial cells and attracts double- and single-positive thymocytes expressing the TECK receptor CCR9. Eur. J. Immunol. 30, 262-271 (2000).

25. Bazan, J.F. et al. A new class of membrane-bound chemokine with a CX3C motif. Nature 385, 640-644 (1997).

26. Niess, J.H. et al. CX3CR1-mediated dendritic cell access to the intestinal lumen and bacterial clearance. Science 307, 254-258 (2005).

27. Hogaboam, C.M. et al. Alteration of the cytokine phenotype in an experimental lung granuloma model by inhibiting nitric oxide. J. Immunol. 159, 5585-5593 (1997).

28. Kanno, M. et al. Functional expression of CCL6 by rat microglia: a possible role of CCL6 in cell-cell communication. J. Neuroimmunol. 167, 72-80 (2005).

29. Brandl, K., Plitas, G., Schnabl, B., DeMatteo, R.P. \& Pamer, E.G. MyD88mediated signals induce the bactericidal lectin Reglll gamma and protect mice against intestinal Listeria monocytogenes infection. J. Exp. Med. 204, 1891-1900 (2007).

30. Bens, M. et al. Transimmortalized mouse intestinal cells ( $\mathrm{m}-\mathrm{ICc} 12)$ that maintain a crypt phenotype. Am. J. Physiol. 270, C1666-C1674 (1996).

31. Nuding, S., Fellermann, K., Wehkamp, J., Mueller, H.A. \& Stange, E.F. A flow cytometric assay to monitor antimicrobial activity of defensins and cationic tissue extracts. J. Microbiol. Methods 65, 335-345 (2006).

32. Yount, N.Y. et al. Structural correlates of antimicrobial efficacy in IL-8 and related human kinocidins. Biochim. Biophys. Acta. 1768, 598-608 (2007)

33. Berahovich, R.D. et al. Proteolytic activation of alternative CCR1 ligands in inflammation. J. Immunol. 174, 7341-7351 (2005).

34. Beninger, P.R. et al. Physical and genetic mapping of the Salmonella dublin virulence plasmid pSDL2. Relationship to plasmids from other Salmonella strains. J. Clin. Invest 81, 1341-1347 (1988).

35. Zhang, X., Kelly, S.M., Bollen, W.S. \& Curtiss, R. III Characterization and immunogenicity of Salmonella typhimurium SL1344 and UK-1 delta crp and delta cdt deletion mutants. Infect. Immun. 65, 5381-5387 (1997).
36. Wick, M.J., Harding, C.V., Normark, S.J. \& Pfeifer, J.D. Parameters that influence the efficiency of processing antigenic epitopes expressed in Salmonella typhimurium. Infect. Immun. 62, 4542-4548 (1994).

37. Gottlieb, C.T. et al. Antimicrobial peptides effectively kill a broad spectrum of Listeria monocytogenes and Staphylococcus aureus strains independently of origin, sub-type, or virulence factor expression. BMC Microbiol. 8, 205 (2008).

38. Youn, B.S. et al. Molecular cloning of leukotactin-1: a novel human beta-chemokine, a chemoattractant for neutrophils, monocytes, and lymphocytes, and a potent agonist at CC chemokine receptors 1 and 3. J. Immunol. 159, 5201-5205 (1997).

39. Pardigol, A. et al. HCC-2, a human chemokine: gene structure, expression pattern, and biological activity. Proc. Natl. Acad. Sci. USA 95, 6308-6313 (1998).

40. Wang, W., Bacon, K.B., Oldham, E.R. \& Schall, T.J. Molecular cloning and functional characterization of human MIP-1 delta, a new C-C chemokine related to mouse CCF-18 and C10. J. Clin. Immunol. 18, 214-222 (1998).

41. Hieshima, K. et al. CC chemokine ligands 25 and 28 play essential roles in intestinal extravasation of IgA antibody-secreting cells. J. Immunol. 173, 3668-3675 (2004).

42. Kunkel, E.J. et al. CCR10 expression is a common feature of circulating and mucosal epithelial tissue lgA Ab-secreting cells. J. Clin. Invest 111, 1001-1010 (2003).

43. Belperio, J.A. et al. Interaction of IL-13 and C10 in the pathogenesis of bleomycin-induced pulmonary fibrosis. Am. J. Respir. Cell Mol. Biol. 27, 419-427 (2002).

44. Wu, Y., Prystowsky, M.B. \& Orlofsky, A. Sustained high-level production of murine chemokine C10 during chronic inflammation. Cytokine 11, 523530 (1999).

45. Kaesler, S., Regenbogen, J., Durka, S., Goppelt, A. \& Werner, S. The healing skin wound: a novel site of action of the chemokine C10. Cytokine 17, 157-163 (2002).

46. Backhed, F., Ley, R.E., Sonnenburg, J.L., Peterson, D.A. \& Gordon, J.I. Host-bacterial mutualism in the human intestine. Science 307, 19151920 (2005).

47. Ghosh, S.K. et al. Quantification of human beta-defensin-2 and -3 in body fluids: application for studies of innate immunity. Clin. Chem. 53, 757-765 (2007).

48. Valore, E.V., Wiley, D.J. \& Ganz, T. Reversible deficiency of antimicrobial polypeptides in bacterial vaginosis. Infect Immun. 74, 5693-5702 (2006).

49. Harder, J., Bartels, J., Christophers, E. \& Schroder, J.M. Isolation and characterization of human beta -defensin-3, a novel human inducible peptide antibiotic. J. Biol. Chem. 276, 5707-5713 (2001).

50. Wu, Z. et al. Engineering disulfide bridges to dissect antimicrobial and chemotactic activities of human beta-defensin 3. Proc. Natl. Acad. Sci. USA 100, 8880-8885 (2003).

51. Schulz-Knappe, P. et al. HCC-1, a novel chemokine from human plasma. J. Exp. Med. 183, 295-299 (1996).

52. Wehkamp, J. et al. Innate immunity and colonic inflammation: enhanced expression of epithelial alpha-defensins. Dig. Dis. Sci. 47, 1349-1355 (2002).

53. Fahlgren, A., Hammarstrom, S., Danielsson, A. \& Hammarstrom, M.L. $\beta$-Defensin-3 and -4 in intestinal epithelial cells display increased mRNA expression in ulcerative colitis. Clin. Exp. Immunol. 137, 379-385 (2004).

54. Nuding, S., Fellermann, K., Wehkamp, J. \& Stange, E.F. Reduced mucosal antimicrobial activity in Crohn's disease of the colon. Gut 56, 1240-1247 (2007).

55. Berger, M.S. et al. The chemokine C10: immunological and functional analysis of the sequence encoded by the novel second exon. Cytokine 8, 439-447 (1996).

56. Asensio, V.C. et al. C10 is a novel chemokine expressed in experimental inflammatory demyelinating disorders that promotes recruitment of macrophages to the central nervous system. Am. J. Pathol. 154, 1181-1191 (1999).

57. Jang, M.H. et al. CCR7 is critically important for migration of dendritic cells in intestinal lamina propria to mesenteric lymph nodes. J. Immunol. 176, 803-810 (2006).

58. Stenstad, H., Svensson, M., Cucak, H., Kotarsky, K. \& Agace, W.W. Differential homing mechanisms regulate regionalized effector CD8alphabeta+ T cell accumulation within the small intestine. Proc. Natl. Acad. Sci. USA 104, 10122-10127 (2007).

59. Meyer-Hoffert, U. et al. Identification of heparin/heparan sulfate interacting protein as a major broad-spectrum antimicrobial protein in lung and small intestine. FASEB. J. 22, 2427-2434 (2008). 